\title{
Holes in illusory conjunctions
}

\author{
LIN CHEN and WU ZHOU \\ University of Science and Technology of China, Beijing, China
}

\begin{abstract}
To address the fundamental question "What are primitives of visual perception?" we tested the hypothesis of early topological perception employing illusory conjunctions. Through a series of experiments, which were well controlled for various feature errors, we consistently observed the illusory conjunction of holes, which are a typical kind of topological property. Subjects in our experiments perceived illusory hollow figures in which the conjoined holes underwent geometric transformations. This indicates that the holes were perceived as abstract topological entities available at an early stage, which provides further support for the hypothesis of topological perception.
\end{abstract}

Treisman and Gelade (1980), among others, have reported that when attention is overloaded, subjects often report seeing illusory conjunctions - that is, incorrect recombinations of the features that they detect. For example, when forced to divide their attention across several briefly presented objects like a red $\mathrm{X}$ and a blue $\mathrm{S}$, subjects often reported perceiving a blue $\mathrm{X}$ or a red S. Owing to its obvious importance, the discovery of illusory conjunctions has stimulated research on numerous issues in the current study of perception and attention, including our own research on the fundamental question "What are the primitives for representing visual information?" Specifically, features that give rise to illusory conjunctions must be separately abstracted at an early visual stage, for if such an abstraction of features did not occur, their illusory conjunctions would be difficult to explain. Thus, features that produce illusory conjunctions are strong candidates for the primitives of visual perception.

The hypothesis of topological perception (Chen, 1982) directly leads to certain candidates for visual primitives. The hypothesis assumes that a primitive and general function of the visual system is the perception of topological properties, including the number of holes. There is evidence that such topological properties serve as primitives in various processes involved in perceptual organization (see, e.g., Chen, 1982, 1985, 1989; Elder \& Zucker, 1993; Han \& Chen, 1994; He \& Chen, 1994; Lan \& Chen, 1996; Zhou \& Chen, 1996). For example, two visual stimuli that are topologically different with respect to holes are more discriminable in a near-threshold same-different task than

We are very grateful to Walt Makous for careful review of the draft We also thank X. Hu for assistance with preparation of these experiments. This research was supported by the State Commission of Science and Technology of China, by a research fellowship from the Alexander von Humboldt Foundation and a grant from Deutsche Forschungsgemeinschaft, Germany, to Professor Ernst Pöppel, and by the Human Frontier Science Program. Part of the results were reported at ARVOs 1992 and 1993. W. Zhou is now affiliated with the Center for Visual Science, University of Rochester. Correspondence should be addressed to L. Chen, Beijing Laboratory of Cognitive Science, Graduate School, Academia Sinica, University of Science and Technology of China, Box 3908, Beijing, China (e-mail: Ichen@public2.bta.net.cn). are other pairs of figures that are topologically equivalent (Figure 1) (Chen, 1990). The discovery of topological structure in vision provides a challenge to computational approaches to vision based on local geometrical properties (see, e.g., Chen, 1989) and, on the other hand, suggests a formal description of Gestalt-type phenomena and Gibsonian invariance perception (e.g., Chen, 1982, 1985).

If topological properties are the basis of visual primitives, illusory conjunctions of topological properties, such as holes, should sometimes occur. To test this prediction, we adopted an experimental paradigm originally designed by Treisman (1986).

\section{EXPERIMENT 1}

\section{Method}

The stimulus displays each contained three different figures (Figure 2). These particular figures were chosen because they are typical examples of a topological difference in holes. That is, the ring in the top row of Figure 2 differs topologically from the solid triangle and ellipse, in that the ring contains a hole whereas the triangle and ellipse do not; although phenomenally they look quite different, the triangle and ellipse are topologically equivalent to each other. As a control for holes, the stimulus display shown in the bottom row of Figure 2, which contained all solid figures, was also added to the stimulus displays. The size of the triangle was $1.4^{\circ} \times 2.7^{\circ}$; the size of the ellipse, $3.4^{\circ} \times 1.4^{\circ}$; and the sizes of the outer and inner circles of the ring were $2.3^{\circ} \times 2.3^{\circ}$ and $1^{\circ} \times$ $1^{\circ}$, respectively. The size of the digits was about $0.4^{\circ} \times 0.7^{\circ}$. And, the width of the three figures and two digits aligned together was about $14^{\circ}$. All the figures and digits were black on a white background.

A three-field projector tachistoscope was used to present the stimuli. The subjects' primary task was to report correctly the two digits flanked on each side. As a secondary task, the subjects were asked to report verbally the shapes of the three figures located at the middle of a display; they were instructed not to guess but to report only what they were fairly confident of seeing. Each test display was followed by a mask consisting of a checkerboard. Exposure durations were adjusted separately for each subject to keep an overall probability of correct responses to shapes at a rate of about $90 \%$. Here, correct responses to shapes refer only to reports of correctly reporting the outline shapes, without considering holes. Errors such as reports of hollow triangle and/or hollow ellipses did not affect the adjustment of exposure durations, for these errors might have been due to illusory conjunctions of holes, the occurrence of which was our primary interest. For the purpose of the present experiment, the locations of the three figures reported were recorded but not considered as a criterion of judging correctness of reports. The mean exposure duration across all the trials was about $90 \mathrm{msec}$, with a range across subjects of about $60-180 \mathrm{msec}$. A set of 114 slides, consisting of 


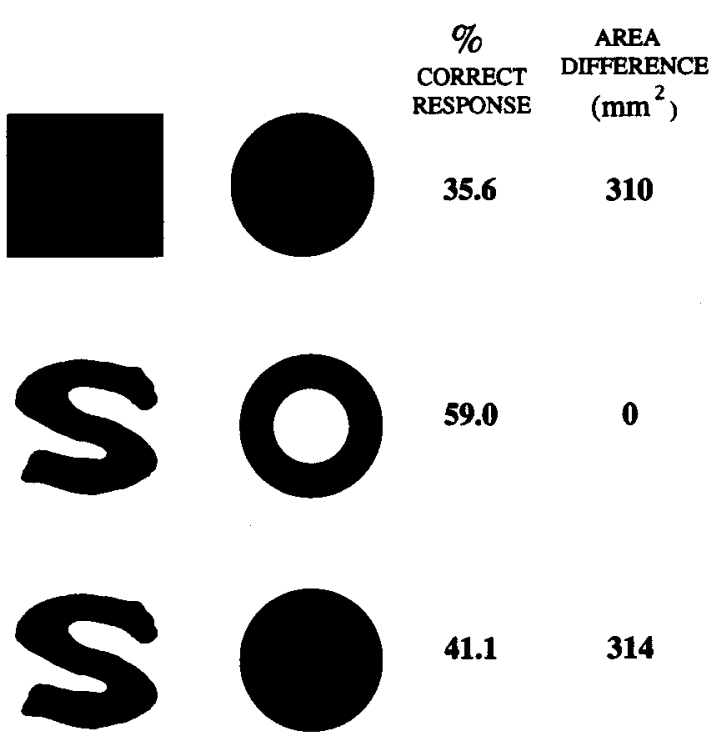

Figure 1. In conditions under which various factors commonly considered in the study of visual perception (e.g., luminous flux, spatial frequency components, and perimeter length) have been controlled, two visual stimuli that are topologically different with respect to holes, such as an S-like figure and a ring, are more discriminable in a near-threshold same-different task than are other pairs of figures that are topologically equivalent, such as a disk and a square or a disk and an S-like figure.
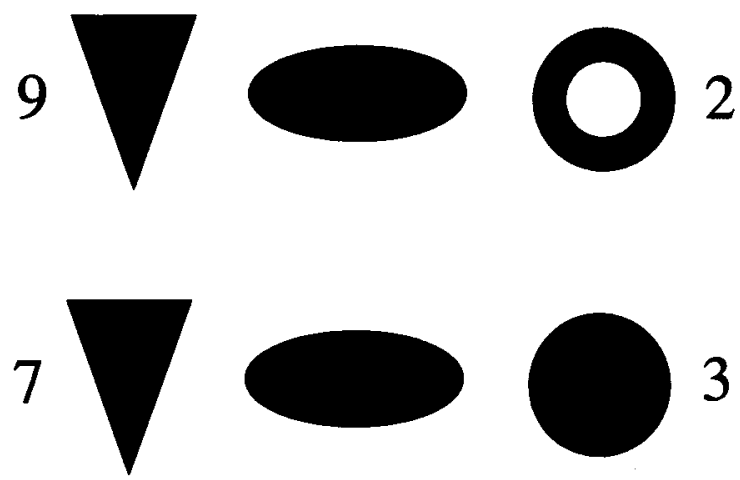

Figure 2. Top: An example of the stimulus displays used in the first experiment. Each contained three different figures-a ring, an ellipse, and a triangle - flanked on each side by a digit. Bottom: An example of the stimulus displays, which, as a control for holes, contained all solid figures.

96 stimuli from the top row of Figure 2 and 18 from the bottom row of Figure 2, as a control, was presented once to each subject. Each figure was presented equally often at each position. Nineteen subjects participated in the experiment.

To examine further the confidence of the subjects in their reports, they were asked to point out which of the figures they had perceived from among a list of various kinds of figures. This was done only in the first two blocks and the last two blocks of presentations. The list of figures that the subjects could point to included, in addition to the four kinds of figures actually presented: two kinds of hollow triangles-a triangle with a triangular hole and a triangle with a circular hole; two kinds of hollow ellipses-an ellipse with an elliptic hole and an ellipse with a circular hole; a solid rectangle; and a solid square.

\section{Results and Discussion}

The results indeed support the prediction of illusory conjunctions of topological features: 15 of 19 subjects (on $16.8 \%$ of all trials) reported seeing hollow triangles and/or hollow ellipses, which were not presented. The topological property of a hole contained by a ring appeared to be abstracted, and then recombined with a solid triangle and/or ellipse so that hollow triangles and/or hollow ellipses were produced. Table 1 summarizes the main aspects of the data.

It might be argued that despite the topological explanation, the hollow ellipses and the hollow triangles that were perceived may have been caused by two kinds of feature errors in perceiving holes; that is, subjects might simply misperceive (1) a solid figure as a hollow one, or (2) a ring as a hollow ellipse or a hollow triangle. The following analysis, leading to the conservative estimate that illusory conjunction of holes occurred on $10.4 \%$ of the trials (shown in Table 1), rules out this objection. The first kind of feature error commonly occurs in the study of illusory conjunctions: subjects report a feature, such as a hole, that is not presented in the stimulus displays. For the present experiment, the rate of such a feature error of holes (the misperception of a solid figure as a hollow one) can be measured by using the stimulus display of Figure 2 (top row), the control for holes: Although the display in this figure contained no hollow figures, subjects reported them. Type 1 of the feature errors of holes in Table 1 shows that the rate of this kind of feature error was $2.2 \%$.

Although the first kind of feature error mentioned above (i.e., to misperceive a feature-a hole in this casethat is not presented), is commonly considered in the study of illusory conjunctions, the second kind of feature error is specific to the present experimental stimuli. The question is: How does one measure this kind of feature error operationally? If subjects misperceive a ring as a hollow ellipse or a hollow triangle - that is, if they misperceive the outer border of the ring as an ellipse or triangle - what

Table 1

Mean Percentage of Correct Reports, Illusory Conjunctions, and Feature Errors of Holes With Verbal Reporting

\begin{tabular}{lc}
\hline \multicolumn{1}{c}{ Response } & \% Total Reports \\
\hline Correct shapes & 88.6 \\
Errors of outline shapes & $11.4^{*}$ \\
Possible illusory conjunctions of holes & 16.8 \\
Feature errors of holes & \\
1. Misperceiving a solid figure as a hollow one & 2.2 \\
2. Misperceiving a ring as a hollow triangle & 2.3 \\
3. Misperceiving a ring as a hollow ellipse & 1.9 \\
Conservative estimate of illusory conjunctions & $10.4 \dagger$ \\
\hline
\end{tabular}

*Under the condition of brief presentation with masking and divided attention, subjects made errors in spontaneously reporting various kinds of outline shapes that were not presented-for example, squares, rectangles, upward triangles or ellipses, repeated shapes such as two disks or two ellipses or two triangles at one presentation, and other irregular shapes. ${ }^{\dagger}$ The difference between illusory conjunctions and feature errors was significantly greater than zero for both the feature errors of Type $1(t$ test, $p<.001)$ and Type $2(t$ test, $p<.001)$, and for the sum of the two types of feature errors ( $t$ test, $p<.002$ ). 
could such reports look like? A direct result would be that it would cause reports of a hollow ellipse or triangle but no ring or disk. Type 2 in Table 1 shows that the second kind of feature error, reports of seeing hollow triangles and/or hollow ellipses without seeing disks or rings, occurred at most on only $4.2 \%$ of the trials. With respect to this type of feature errors, a report was counted as an illusory conjunction only if the subject reported seeing one of the sets of figures illustrated in Figure 3. Otherwise, to keep the estimate of illusory conjunctions conservative, reports of hollow triangles and hollow ellipses, illustrated in Figure 4, were not counted as illusory conjunctions. Instead, the reports were attributed to Type 2 of feature errors of holes in Table 1, even though they might in fact have been produced by illusory conjunctions. (That is, an illusory conjunction of holes actually occurred, but an error in perception of shape also occurred-namely, the misperception of a disk as another shape.) Thus, at least the excess of conjunction errors over the sum of these two types of feature errors, $10.4 \%[(16.8-2.2-4.2) \%]$, represents genuine incorrect combinations of holes rather than simply misperceptions of holes. [Because the feature errors of outline shape were kept below $11.4 \%=(100-$ $88.6) \%$, and because the feature errors of perceiving a ring as a hollow triangle or a hollow ellipse occurred at a rate
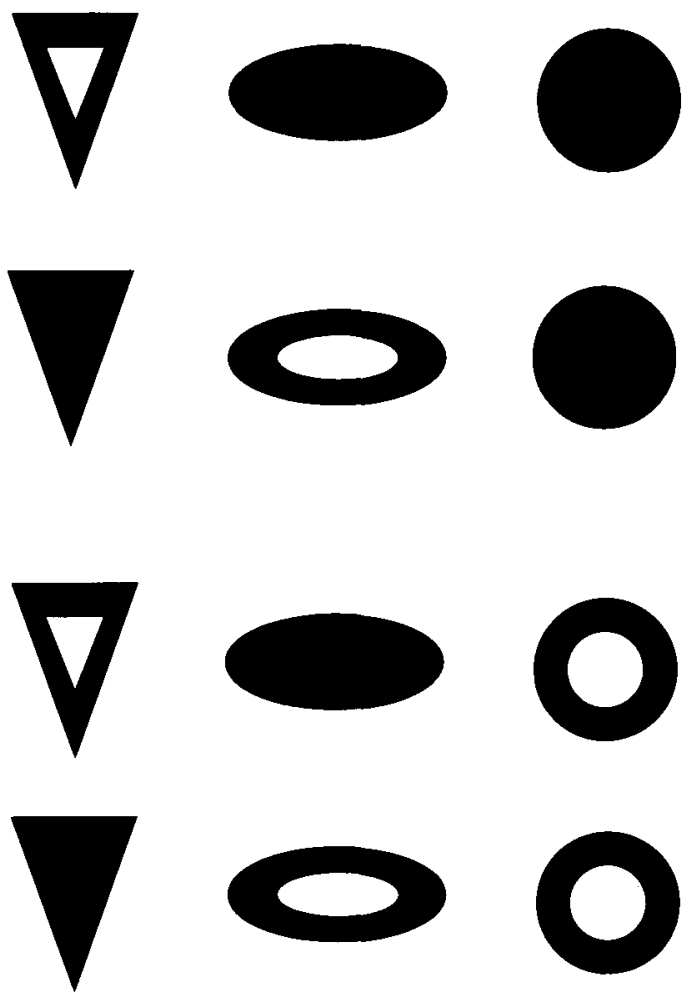

Figure 3. A report was counted as an illusory conjunction only if the subject reported seeing a hollow triangle or a hollow ellipse and, at the same time, a solid disk (top two rows), or a hollow triangle and/or a hollow ellipse and, at the same time, a ring (bottom two rows).
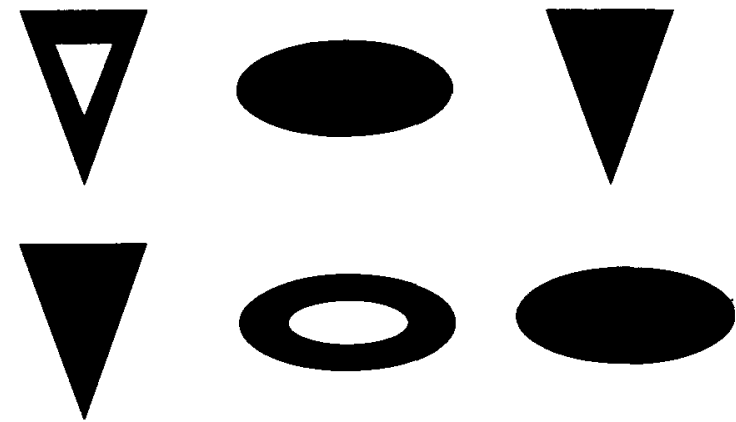

Figure 4. Reports of hollow triangles and hollow ellipses other than those in Figure 3 were not counted as illusory conjunctions. Instead, the reports were attributed to Type 2 feature errors of holes (see Table 1).

of less than $4.2 \%$, the probability that the two kinds of feature errors would occur on the same trial was at least much lower than $4.2 \%$, so they were omitted.]

With respect to reporting by pointing, the results further support the argument that the conjunction errors involving holes are genuine perceptual experiences in the following two senses. First, subjects showed confidence in seeing illusory conjunctions of holes: 17 subjects, including 2 who did not verbally report seeing hollow triangles or ellipses, pointed out hollow triangles and/or ellipses at a rate of $21 \%$ of the total presentations; but only 3 subjects pointed out solid rectangles and/or squares, with an average rate of less than $1 \%$. Second, it is particularly interesting that only triangles containing a triangular hole and ellipses containing an elliptic hole were reported by pointing; in other words, no subjects reported seeing triangles containing a circular hole and/or ellipses containing a circular hole. If the subjects simply misperceived the outline shape of the ring or simply superimposed the circular hole contained by the ring on the solid figures because of, say, afterimages, it would be difficult to explain why no circular holes with triangles or ellipses were perceived. This aspect of the result also indicates the abstract nature of the holes contained in the illusory hollow figures; that is, the concrete shape of a hole is irrelevant to its identity as a hole. The abstract nature suggests that holes were extracted before their illusory conjunctions.

\section{EXPERIMENT 2}

In Experiment 1, 8 subjects who reported seeing hollow triangles and/or hollow ellipses by pointing said at that time that in their verbal reports they often did not distinguish between a hollow figure and a solid one of the same outline shape. This aspect of the results calls attention to a limitation on the verbal reporting of shapes. On the other hand, to instruct subjects to report particularly if there are holes might introduce the possibility of bias. In view of these considerations, and as a further test of illusory conjunctions of holes, in the second experi- 
ment, we used a different procedure for reportingnamely, drawing by hand instead of reporting verbally.

\section{Method}

The method was identical to that of Experiment 1 except that subjects reported via drawing by hand instead of reporting verbally. For the first two blocks of presentations, the subjects were asked to report what they saw also by pointing among the same list of figures as in the first experiment, and feedback was given only if a subject reported a hollow figure by pointing but drew it as solid. Nine subjects who had not participated in Experiment 1 took part in the second experiment

\section{Results and Discussion}

As can be seen in Table 2, the results showed illusory conjunctions on $17.6 \%$ of the trials ( $t$ test, $p<.001)$, much higher than those shown by verbal report in the previous experiment. The converging results, obtained by the different procedure of reporting by hand drawing, further confirm the illusory conjunction of holes.

\section{EXPERIMENT 3}

One distinguishing aspect of illusory hollow figures produced in Experiments 1 and 2 is that the conjoined holes underwent remarkable geometric transformations, such as shape transformations of a circle to a triangle or to an ellipse. The next question is, Which constraints, if any, play a role in such drastic deformations shown by illusory holes? One more experiment was run to examine the constraints.

\section{Method}

A new sample of 8 subjects participated in Experiment 3. In a procedure similar to that used in Experiment 2, subjects were shown a set of three outline figures (see Figure 5). These stimuli were used to represent the inside/outside relationship, another kind of topological invariant. Although deformations restricted to the spatial dimensions of the figures could change the shapes and locations of the figures, such deformations could not change the inside/outside relationship between the figures; that is, such deformations could not change which objects were located inside and which were located outside the circle, for example. The size of the outline triangle was $3^{\circ} \times 4^{\circ}$; the size of the outline square, $4^{\circ} \times 4^{\circ}$; and the sizes of the outer outline circle and inner circle, were $4^{\circ} \times 4^{\circ}$ and $1^{\circ} \times 1^{\circ}$, respectively. The size of the digit letters was about $1^{\circ} \times 1^{\circ}$. The width of the three figures and two digits aligned

Table 2

Mean Percentage of Correct Reports, Illusory Conjunctions, and Feature Errors of Holes With Reporting by Hand Drawing

\begin{tabular}{lc}
\hline \multicolumn{1}{c}{ Response } & \% Total Reports \\
\hline Correct shapes & 90.6 \\
Errors of outline shapes & 9.4 \\
Possible illusory conjunctions of holes & 24.0 \\
Feature errors of holes & \\
$\quad$ 1. Misperceiving a solid figure as a hollow one & 1.8 \\
2A. Reporting a hollow triangle but without & \\
$\quad$ a ring or disk & 2.2 \\
2B. Reporting a hollow ellipse but without & \\
$\quad$ a ring or disk & 2.4 \\
Conservative estimate of illusory conjunctions & $17.6^{*}$
\end{tabular}

*The difference between illusory conjunctions and feature errors was significantly greater than zero for the feature errors of both Type 1 ( $t$ test, $p<.001$ ) and Type 2 ( $t$ test, $p<.001$ ), and for the sum of the two types of feature errors ( $t$ test, $p<.001$ ).
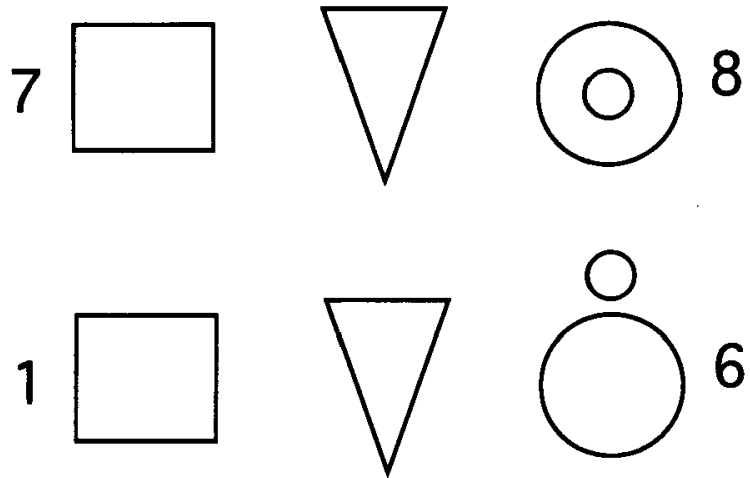

Figure 5. Top: stimulus displays representing the inside relationship. Bottom: stimulus displays used, as a control for the inside/ outside relationship, to represent the outside relationship.

together was about $20^{\circ}$. All the figures and digits were black on a white background.

\section{Results and Discussion}

When shown the stimuli in the top row of Figure 5, all subjects reported seeing a small figure contained within a larger triangle or square, and when shown the stimuli in the bottom row of Figure 5, all saw a small figure outside a larger triangle or square. To keep the estimate of illusory conjunction conservative, only the response sets in Figure 6 were considered illusory conjunctions. They occurred on $16.6 \%$ ( $t$ test, $p<.001$ ) and $12.4 \%$ ( $t$ test, $p<$ .002 ), respectively, of the total presentations of the stimuli in Figure 5.

The following points are noteworthy. (1) With the stimuli of Figure 5 (top), the same shape transformation as that in Experiment 1 was observed. All subjects except one (who reported twice seeing a small circle contained inside a larger square) reported seeing only a small triangle and square, enclosed by a larger triangle and square, respectively, rather than the small circle that was actually presented. (2) With the stimuli in the top row of Figure 5 , no subject reported seeing a small figure outside a larger one; with the stimuli in the bottom row of Figure 5 , no subject reported a small figure encircled by a larger one either. This indicates that although transformations of shape occur in the production of illusory conjunctions, two topological constraints - those of number of holes and the inside/outside relationship - remain invariant in the illusory conjunctions.

\section{GENERAL DISCUSSION}

The illusory conjunctions of holes reported here confirm the validity of the phenomenon of illusory conjunctions. Furthermore, the illusory conjunction of holes provides robust support to the hypothesis of the early topological perception.

A major difficulty with the evidence for the early topological perception is that it is difficult to design stimuli that represent only topological distinctions without introducing differences in nontopological variables, such as spatial frequency components or luminous flux, that are commonly considered in the study of visual perception. Even though 

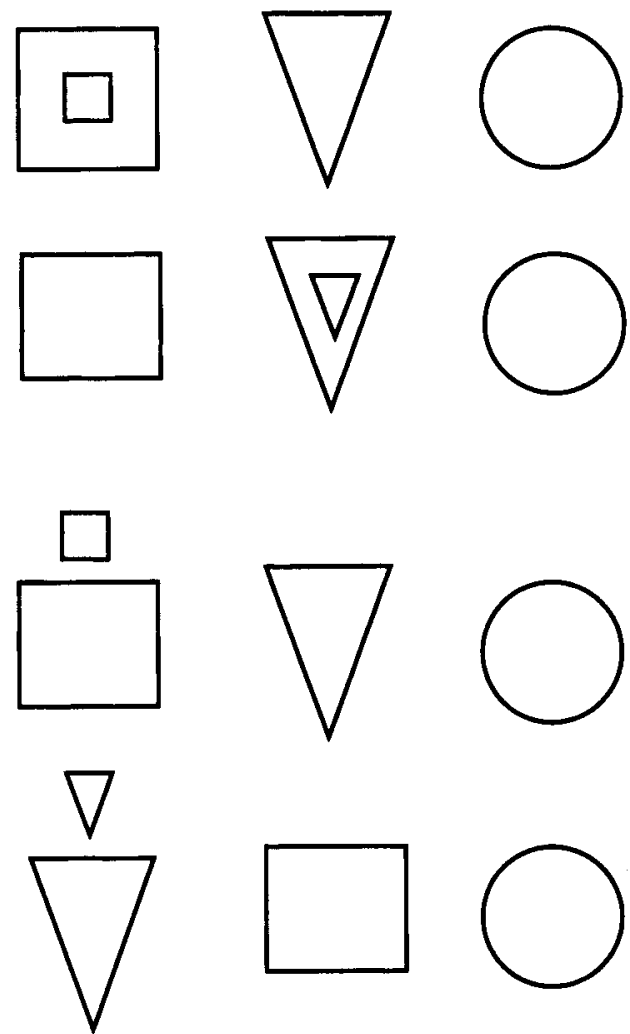

Figure 6. Examples of the response sets that were considered to be illusory conjunctions. To keep the estimate of illusory conjunction conservative, only these response sets were counted.

various factors (e.g., luminous flux, spatial frequency components, and perimeter length) may be controlled under conditions, such as those in Figure 1, a single piece of evidence for topological perception still leaves room for an argument against the topological hypothesis in terms of confounding by nontopological factors (e.g., for texture perception, see Julesz, 1981; for visual sensitivity, see Rubin \& Kanwisher, 1985; for visual search, see Treisman \& Gormican, 1988). This confounding seemed unavoidable before discovery of the illusory conjunction of holes. However, the holes in illusory conjunctions differ from those in the original stimuli (e.g., in shape), and they do not depend on detailed geometric properties or physical properties such as spatial frequency components and luminous flux. It is therefore quite difficult to explain these results in terms of confounds of such nontopological features.

\section{REFERENCES}

CHEN, L. (1982). Topological structure in visual perception. Science, 218, 699-700.

CHEN, L. (1985). Topological structure in the perception of apparent motion. Perception, 14, 197-208.

ChEN, L. (1989). Topological perception: A challenge to computational approaches to vision. In R. Pfeifer, Z. Schreter, E. Fogelman-Soulie, \& L. Steels (Eds.), Connectionism in perspective (pp. 317-329). Amsterdam: Elsevier.

CHEN, L. (1990). Wholes and holes: A reply to Rubin and Kanwisher. Perception \& Psychophysics, 47, 47-53.

ELDER, J., \& ZUCKER, S. (1993). The effect of contour closure on the rapid discrimination of two-dimensional shapes. Vision Research, 33, 981-991.

HAN, S. H., \& ChEN, L. (1994). A topological approach to global precedence. Investigative Ophthalmology \& Visual Science, 35(Suppl.), 1625 .

HE, D. H., \& CHEN, L. (1994). Topological illusory conjunctions: The inside/outside relationship. Investigative Ophthalmology \& Visual Science, 35(Suppl.), 1622.

JULESZ, B. (1981). Textons, the elements of texture perception, and their interactions. Nature, 290, 91-97.

LAN, Z., \& CHEN, L. (1996). Invariance perception approach to the study of hemispheric asymmetry: Topological RVF/left hemispheric superiority. Investigative Ophthalmology \& Visual Science, 37, S170.

Rubin, J., \& KanWISher, N. (1985). Topological perception: Holes in an experiment. Perception \& Psychophysics, 37, 179-180.

Treisman, A. [M.] (1986, November). Features and objects in visual processing. Scientific American, 255 (5), 114B-125.

Treisman, A. M., \& Gelade, G. (1980). A feature-integration theory of attention. Cognitive Psychology, 12, 97-136.

Treisman, A. [M.], \& GoRMican, S. (1988). Feature analysis in early vision: Evidence from search asymmetries. Psychological Review, 95, $15-48$.

ZHou, T. G., \& CHEN, L. (1996). Hierarchy of perceptual primitives: Evidence from search rates. Investigative Ophthalmology \& Visual Science, 37, S296.

(Manuscript received June 30, 1995; revision accepted for publication May 22, 1997.) 